\section{In the beginning}

John Gerhart

Embryos: Color Atlas of Development. Edited by Jonathan B. L. Bard. Wolfe: 1994. Pp. 224. £49.95.

As Jonathan Bard explains in his introduction, the discovery of a host of molecules shared by a wide variety of developing organisms has been the first reward for those analysing the molecular genetics of developmental processes. This has stimulated interest in comparative development as an inquiry into the shared molecular mechanisms of development. This book was prepared to aid such studies, not by summarizing the results of molecular developmental research but by providing background information on the development of 12 organisms commonly used in research, each covered in a chapter by an author well known for research in the field: Arabidopsis (the single plant example), Dictyostelium (a slime mould, not an embryo), sea urchin, nematode worm (Caenorhabditis elegans), molluscs, leech (annelid), Drosophila (insect), zebrafish, Xenopus and other amphibia, chick, mouse and human. The text of each chapter contains a description of the stages of development; an outline of advantages the organism holds for experimental studies, such as the analysis of mutants blocked in various aspects of development; a brief mention of the status of molecular analysis; a forecast of the organism's place in future developmental studies; and key references. All chapters are well illustrated by colour plates and diagrams (600 colour plates in total).

This is a valuable compendium for all students of development. Undergraduate or graduate students can use it with a separate text, and researchers can use it with texts and research articles. Many of the illustrations are valuable for teaching The colour plates are of high quality, and the authors have made an effort to collect excellent material from their research colleagues and to present their own photographs. It is a pleasure just to leaf through the book and look at the pictures.

Beyond increasing comprehension, colour figures make some central phenomena of development inescapably apparent in ways they weren't before. All readers will be struck by region-specific gene expression as revealed by the new staining techniques involving in situ hybridization, lac $Z$ reporter constructs and multicolour antibodies. A set of colour plates of regional gene expression at successive stages of an embryo's development reveals a strikingly dynamic and complex anatomy, one hardly appreciable by simply looking down a microscope. Still the wellspring of regionally expressed test molecules, Drosophila provides the most impressive pictures, but the techniques have spread to other organisms, such as nematodes, sea urchins and recently amphibia and mice. It is to be hoped that a second edition of the atlas will follow in a few years when comparable in situ and antibody pictures are available for these organisms as well.

John Gerhart is in the Department of Molecular Biology, University of California, Berkeley, California 94720-3200, USA.

\section{Geo-exploration}

\section{David Gubbins}

Earth Sounding Analysis: Processing versus Inversion. By Jon F. Claerbout. Blackwell Scientific: 1992. Pp. 304. £45. Latest CD-ROM version available from $J$. F. Claerbout, Department of Geophysics, Stanford University, Stanford, California 94305-2215, USA.

THE Hitch Hiker's Guide to the Galaxy is a fictional electronic book. It resembles a notebook computer, provides useful information for galactic travellers and has DON'T PANIC on the front. Earth Soundings Analysis is a real electronic book: in addition to a paper edition published some time ago, there are more recent $C D$ versions that promise interactive diagrams, machine-readable text and computer programs. It is an imaginative step forward in scientific publishing, despite being a highly specialist work.

Claerbout is an innovative leader in seismic-processing research, through the Stanford Exploration Project. His style is pleasantly casual. The first four chapters give essential background mathematics and the remaining chapters concentrate on seismic-processing methods. Relegating statistics to the final chapters will bother statisticians but is probably how seismic processing should be taught

The material is difficult but rewarding. Particularly useful are the conventional discussion of the conjugate gradient methods, the innovative and intriguing treatment of missing data and the chapter on minimum phase. I did not fully understand the chapter on conjugate operators. Although this subject comprises an important underlying philosophy, it needs more mathematical development and structure, Claerbout, like most exploration geophysicists, has a very narrow definition of 'inversion' - least-squares modelling. Perhaps for this reason or perhaps because I did not understand the message on conjugate operators, the 'versus' between inversion and processing is not obvious. I expected a contrast between optimization and filtering methods, but the only clear contrast in the book is between practical and impractical methods.

The electronic version contains both the book and PhD projects. Claerbout clearly believes his product goes beyond simply electronic reproduction of text and computer programs; it can be used to reproduce the calculations done during the research, to rebuild datasets and programs and to extend applications to new problems. He uses the term "reproducible research", by which he means the publication of working programs and data as an integral part of a scientific report, made possible by the electronic revolution. It is different from 'reproducibility of science', where a scientific result is described in sufficient detail and with sufficient accuracy for it to be repeated by others.

For example, scientific reproducibility allows us to test Newton's law of gravitation to far greater accuracy than could seventeenth-century astronomers. The result is strengthened if confirmed, or gives way to a new theory (general relativity in this case) if some discrepancy is found. This scientific process is different from repeating Tycho Brahe's observations with the same instrumentation: we might want to do this to clarify our understanding of the original procedures, but the results would add nothing to science. Claerbout is concerned with this latter, more practical goal: a worthy one in view of the lax nature of many modern scientific papers and the dependence of the results on bug-prone computer programs.

As well as the CD-ROM drive, the electronic book needs 15 megabytes of hard-disk space and creates hundreds of irritating files. It gives access to all the text and an enormous volume of exploration geophysics software. Few of the diagrams are interactive, and those that are depend on just two different programs. Unfortunately, the CD cannot be used over the network, and it took a long time on our machines (Sun 4) to draw any of the diagrams.

At Leeds we teach seismic processing and inversion using a program called XPITSA, available through the International Association of Seismology and Physics of the Earth's Interior. It is menudriven (and therefore quick for students to learn), occupies little disk space, and encompasses far more of the subject than the interactive diagrams in Claerbout's book.

Claerbout's important contribution to the electronic publishing revolution is to put together publications with programs and data, as an essential new type of scientific publication. Not quite The Hitch Hiker's Guide, perhaps, but a big change in the way we learn and do science.

David Gubbins is in the Department of Earth Sciences, University of Leeds, Leeds LS2 9JT, UK 